\title{
Nuclear imaging for diagnosing fracture-related infection
}

\author{
Paul Bosch ${ }^{1}$ - Andor W. J. M. Glaudemans ${ }^{2}$. Jean-Paul P. M. de Vries ${ }^{1} \cdot$ Tim R. Middelberg $^{3} \cdot$ Geertje A. M. Govaert $^{4}$. \\ Frank F. A. IJpma ${ }^{1}$
}

Received: 8 March 2020 / Accepted: 23 May 2020 / Published online: 10 June 2020

(c) The Author(s) 2020

\begin{abstract}
Purpose Fracture-related infection (FRI) is a complication of surgical fracture treatment and can be challenging to diagnose. Recent studies show promising results for the use of either FDG-PET/CT or WBC/anti-granulocyte scintigraphy. The purpose of this pictorial essay is to outline recent developments in nuclear imaging techniques to diagnose FRI.

Methods The current literature on this topic is reviewed. Additionally, three examples of patients who underwent nuclear imaging as part of their clinical work-up and surgical treatment for FRI are presented.

Results Based on recent retrospective studies, FDG-PET/CT (accuracy 0.83) and WBC scintigraphy with SPECT/CT (accuracy 0.92 ) both have a good diagnostic accuracy for diagnosing fracture-related infection. Nuclear imaging contributes to a correct diagnosis in patients with FRI.

Conclusion Retrospective studies show promising results for both FDG-PET/CT and WBC scintigraphy with SPECT/CT in diagnosing FRI. A prospective, multicenter study (IFI trial), directly comparing MRI, FDG-PET/CT, and WBC scintigraphy with SPECT/CT in patients with suspected FRI, is currently in progress.
\end{abstract}

Keywords Fracture-related infection · FRI $\cdot$ Infection $\cdot$ FDG-PET/CT $\cdot$ WBC scintigraphy $\cdot$ Fracture

\section{Introduction}

Fracture-related infection (FRI) is a serious complication that may occur after surgical fracture treatment. It often results in the need for long-term antibiotic therapy, multiple surgical reinterventions, and substantial morbidity [1-3]. The incidence of infection after fracture treatment varies between 1 and 45\%, depending on patient (age, comorbidity, and medication) and fracture (location, contamination, and soft-tissue damage) characteristics $[4,5]$. Its clinical

Paul Bosch

Paulbosch87@gmail.com

1 Department of Surgery, University of Groningen, University Medical Center Groningen, Groningen, The Netherlands

2 Department of Nuclear Medicine and Molecular Imaging, University of Groningen, University Medical Center Groningen, Groningen, The Netherlands

3 Department of Plastic Surgery, University of Groningen, University Medical Center Groningen, Groningen, The Netherlands

4 Department of Surgery, Utrecht University, University Medical Center Utrecht, Utrecht, The Netherlands presentation is heterogeneous, and a timely diagnosis is essential for successful treatment $[6,7]$. Several studies reported on diagnostic and treatment strategies for infection after fracture surgery over the last decade, though the lack of a standardized definition has made direct comparison between studies difficult. Fortunately, a standardized definition for infection after fracture surgery was introduced recently.

In 2018, a consensus definition for FRI was published in collaboration between the AO Foundation and the European Bone and Joint Infection Society (EBJIS) in which the diagnostic strategy for FRI was outlined [8]. The term 'fracture-related infection' is considered to encompass the complete spectrum of infections (e.g., acute and chronic, superficial and deep, with and without bone involvement, with and without implants in situ) following surgical fixation of closed or open fractures. Symptoms can be either confirmatory or suggestive for the presence of FRI. Confirmatory criteria (FRI definitely present) are either the presence of a fistula or wound breakdown, two (out of five) positive microbiology results of intra-operative deep tissue cultures, or histological positive ( $>5$ neutrophils per high powered 
field) results. Suggestive criteria (FRI possibly present) consist of elevated serum inflammatory markers, clinical signs of infection, one (out of five) positive culture results, or positive results of medical imaging studies. It is, however, not defined in this consensus definition paper which imaging technique should be used.

The aim of this paper is to outline the role of nuclear imaging in the diagnostic work-up for FRI and also optimizing FRI treatment by assessing the extent of the infection. The current literature is reviewed, and three clinical cases are presented with several images of both the diagnostic process and surgical treatment.

\section{Imaging modalities in FRI}

Conventional imaging modalities such as radiography and computed tomography (CT) can be used to detect secondary signs of infection such as delayed- or non-union, bone lysis and implant failure (e.g., breakage of plates, nails, or screws). These signs lack specificity, since all can be present in the absence of infection, such as aseptic non-union due to mechanical instability or insufficient perfusion of the tissue. Conventional imaging modalities do help the surgeon to assess fracture healing to decide whether the potentially infected implant can be safely removed or not. Magnetic Resonance Imaging (MRI) has a better ability to identify secondary signs of infection in the soft tissues, such as sinus tracts and abscesses [9]. Important limitations of MRI in the setting of FRI are the scattering caused by metallic implants at the fracture site [10] and the inability to differentiate between infection and inflammation [11].

Nuclear imaging techniques have been used in infectious bone disease for several decades. Bone scintigraphy (BS) shows good sensitivity for FRI, but strongly lacks in specificity making it unsuitable for diagnosing FRI [12].

\section{The role of FDG-PET/CT and WBC/ anti-granulocyte scintigraphy}

Studies on the role of WBC or anti-granulocyte scintigraphy and FDG-PET/CT in bone and joint infection have been performed extensively; however, heterogeneity in both the definition of infection and the imaging protocols used makes comparison between these studies difficult [11]. The most recent EANM guideline for the use of FDG-PET/CT in inflammation and infection states that the evidence for the use of FDG-PET in 'osteomyelitis' of any origin remains low, and that WBC or anti-granulocyte scintigraphy is the preferred imaging modality [13]. However, these guidelines are based on studies regarding peripheral osteomyelitis of any origin, and not specifically in the post-traumatic setting. Our three cases (Figs. 1, 2, 3, 4, 5, 6, 7, 8 and 9) demonstrate that both FDG-PET/CT and WBC scintigraphy can be used in the diagnostic work-up of FRI. Moreover, all three cases illustrate the importance of accurate diagnostic imaging, because an FRI usually has a large clinical and personal impact on the patient.

In 2018, a study assessing the diagnostic accuracy of FDG-PET/CT in 135 patients with suspected FRI was published. Both qualitative (visual) and semi-quantitative [standardized uptake value (SUV) in volume of interest (VOI)] analyses were performed [14]. The consensus definition of FRI was used, and current EANM imaging protocols were adhered to. Visual analysis alone showed an $89 \%$ sensitivity and an $80 \%$ specificity for detecting FRI, with a diagnostic accuracy of $83 \%$. Combining visual and semi-quantitative analysis resulted in a slight increase in diagnostic accuracy up to $86 \%$. This study also showed a sharp increase in false-positive results in the immediate post-surgical setting $(<4$ weeks after fracture fixation), most likely caused by inflammation due to soft-tissue regeneration and bone formation after surgical treatment. This underlines an important limitation of FDG-PET/CT in the early postsurgical setting. Another 2019 study assessed the diagnostic
Fig. 1 Case 1, Clinical presentation
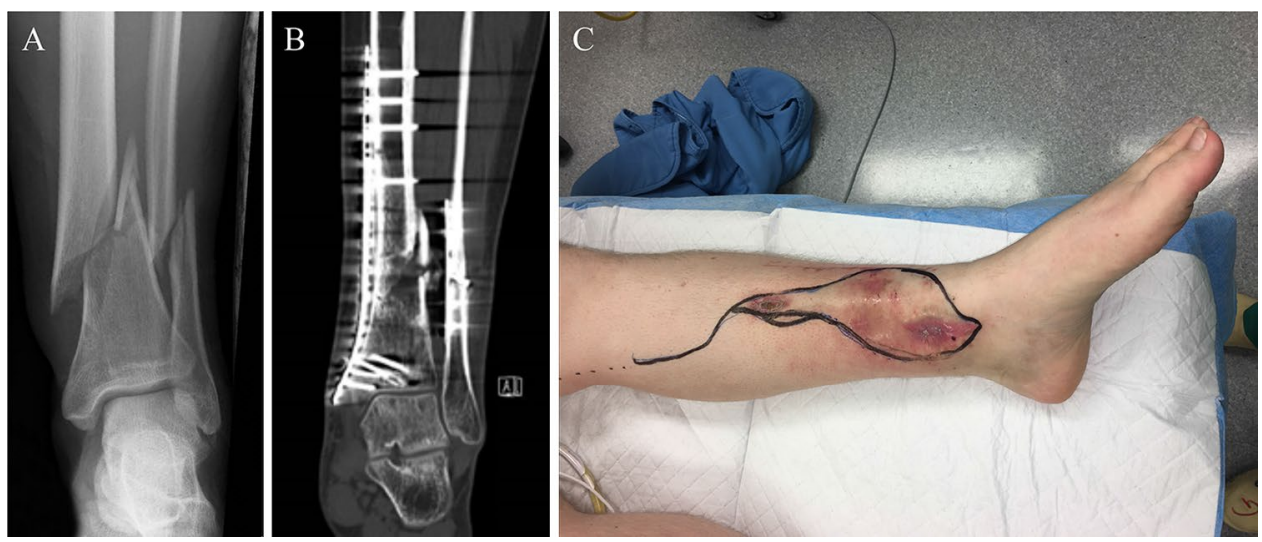
Fig. 2 Case 1, FDG-PET and WBC scintigraphy
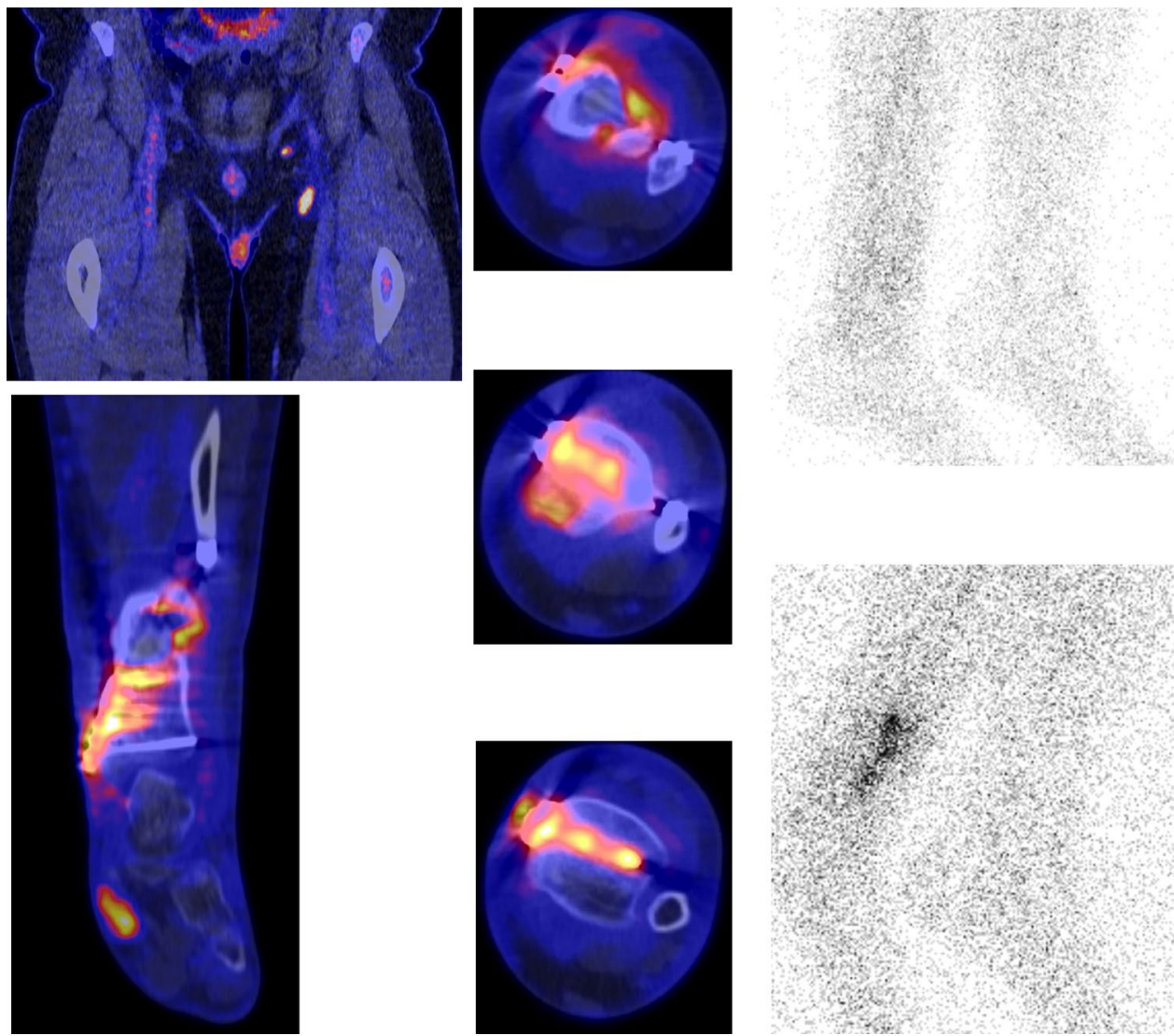

Fig. 3 Case 1, Masquelet procedure stage 1
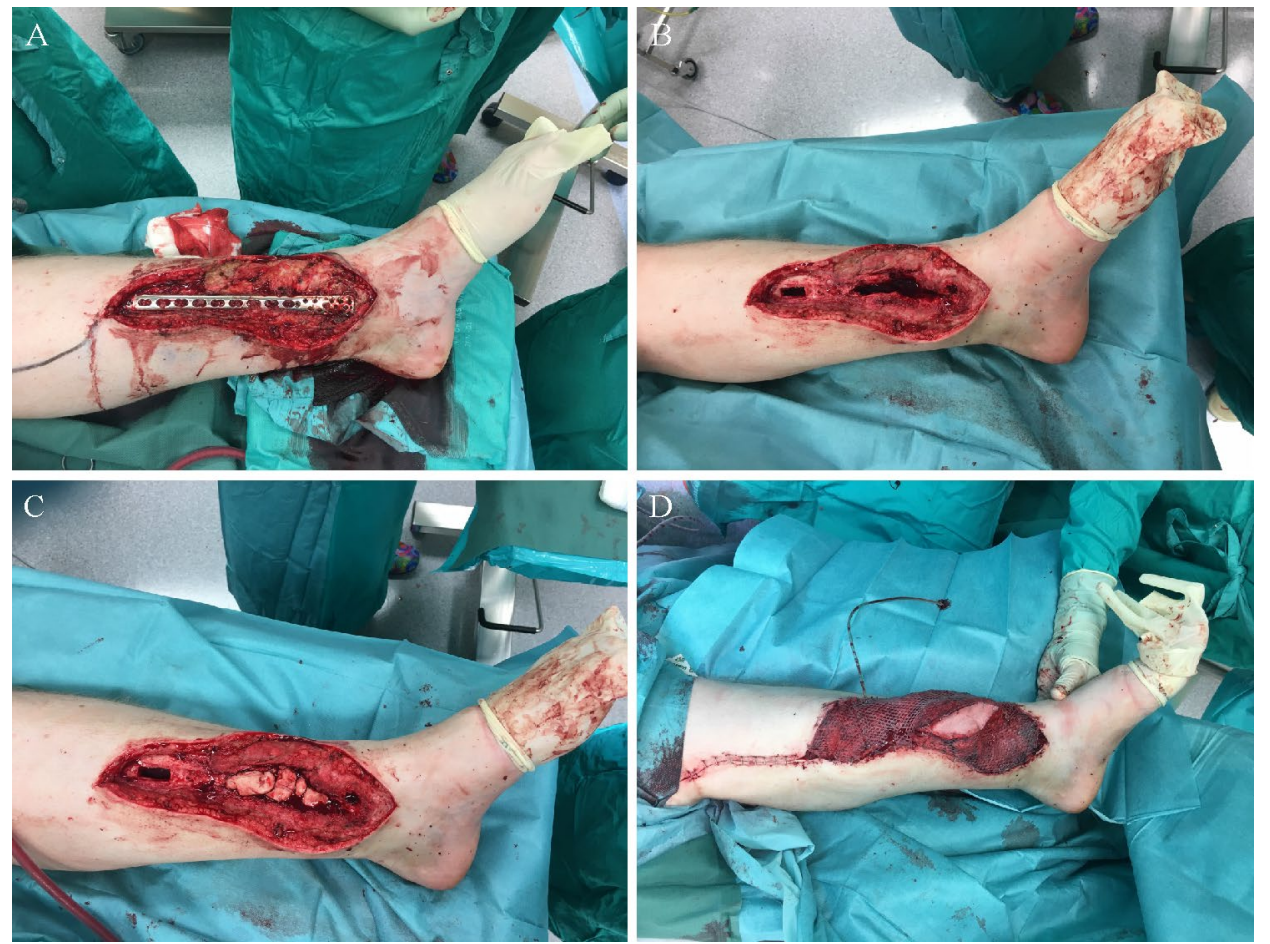
Fig. 4 Case 1, Masquelet procedure stage 2
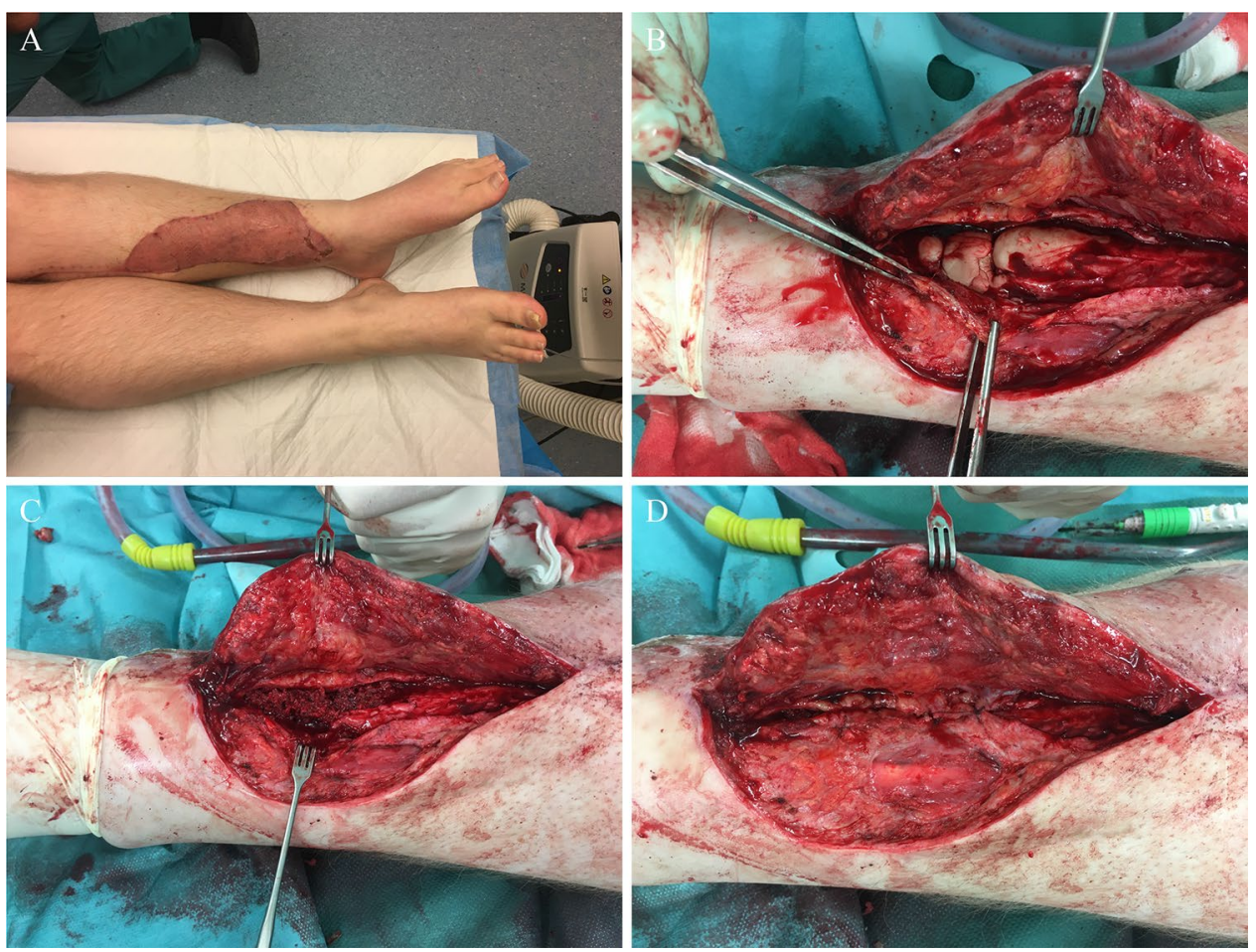

Fig. 5 Case 1, Follow up

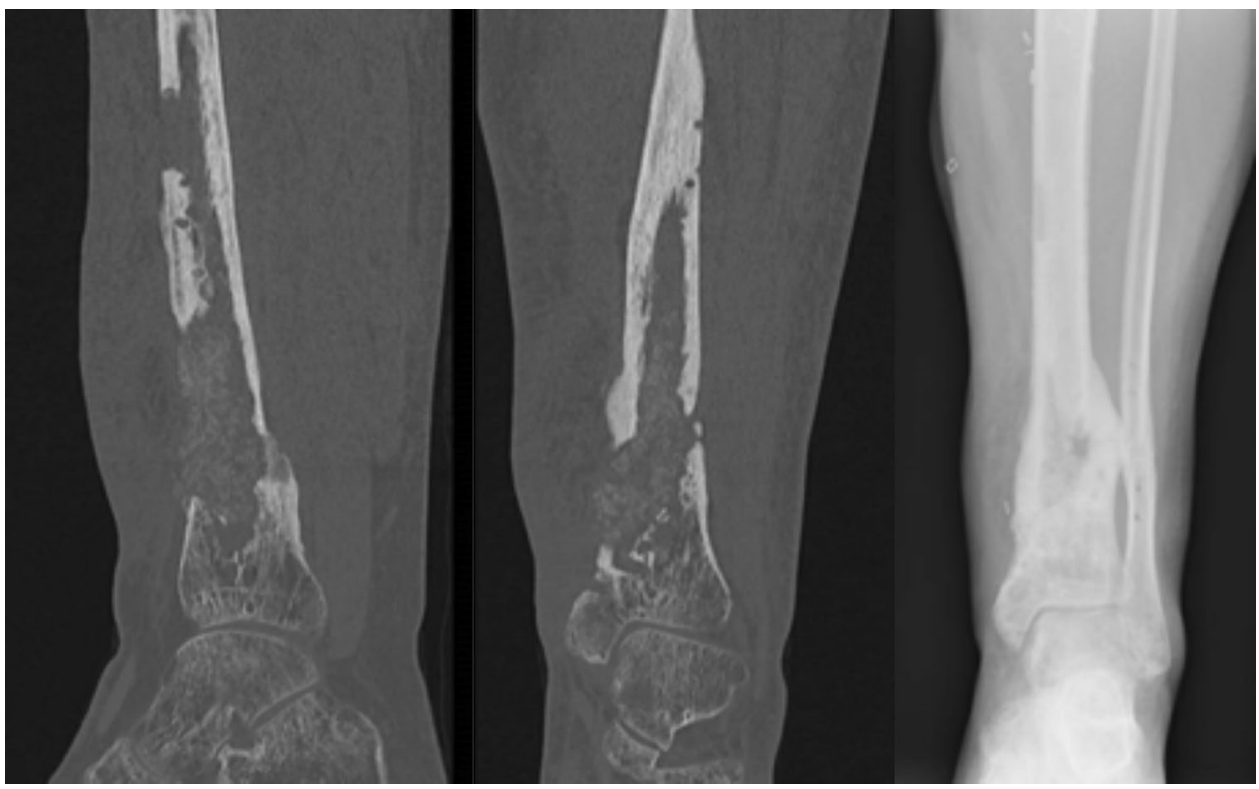

accuracy of FDG-PET/CT in diagnosing infection in patients with non-united fractures [15]. Peri-operative deep tissue cultures were used as the golden standard. They reported a diagnostic accuracy of $81 \%$, which is in line with the results reported in the aforementioned study.

A 2018 retrospective cohort study on the diagnostic accuracy of WBC scintigraphy with SPECT/CT in 192 patients with suspected FRI found a sensitivity of 0.79 and a specificity of 0.97 , with a diagnostic accuracy of 0.92 [16].
The current EANM guidelines were adhered, and infection diagnosis was based on the current FRI consensus definition criteria (per-operative cultures or a minimum of 6 months clinical follow-up). Accuracy was not influenced by recent surgery. Beside radio-labelled autologous white blood cells, scintigraphy using radio-labeled anti-granulocyte antibodies can also be used to diagnose bone infections in the posttraumatic setting. A 2004 cohort study assessed the accuracy of anti-granulocyte scintigraphy in diagnosing bone-related 
Fig. 6 Case 2, Clinical presentation
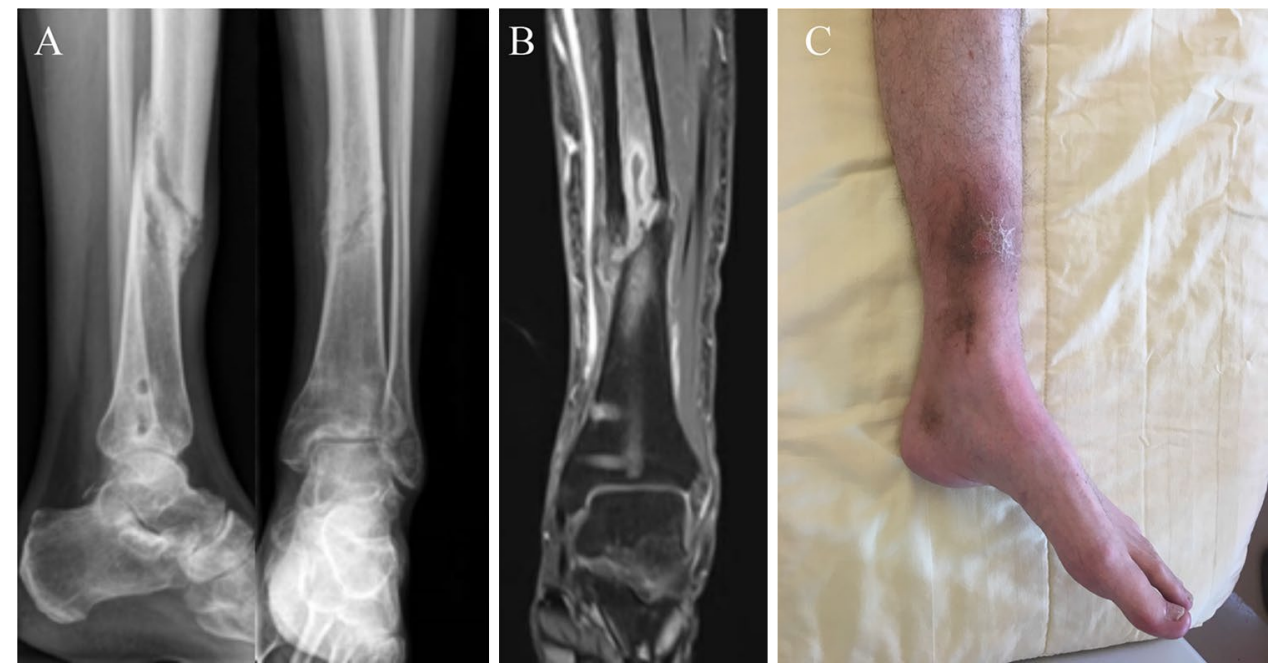
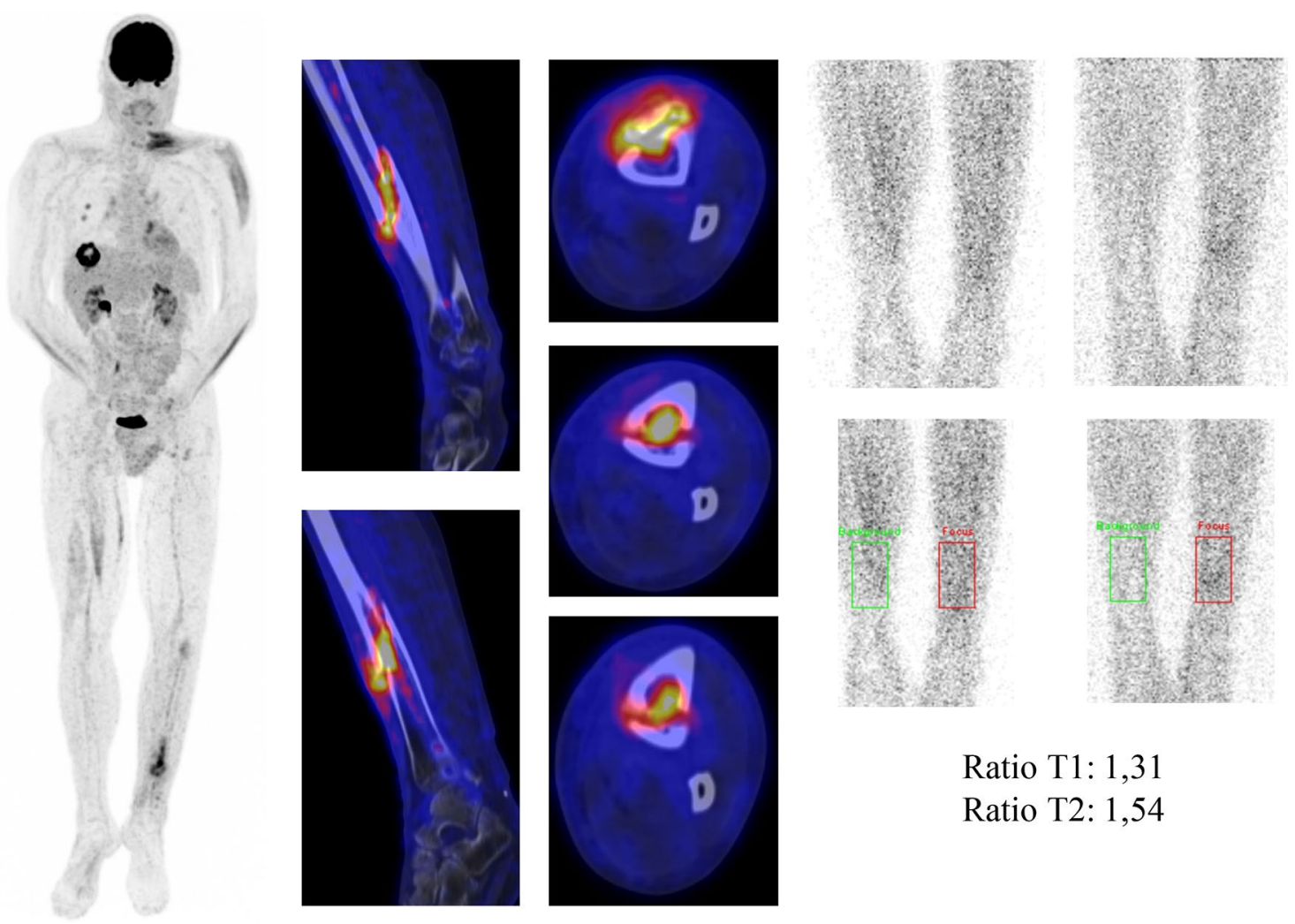

Ratio T1: 1,31

Ratio T2: 1,54

Fig. 7 Case 2, FDG-PET and WBC scintigraphy

infection in 220 patients. The study population consisted of patients with a diabetic foot, joint prosthesis, osteosynthesis after fractures, and spondylodiscitis. They clustered the prosthesis and osteosynthesis groups, and found a sensitivity of $84.2 \%$ and a specificity of $85.7 \%$ [17]. They used a dual time-point imaging protocol $(4 \mathrm{~h}$ and $24 \mathrm{~h}$ after antigranulocyte injection), but the patient population is too heterogenous for the results to be translated to the FRI setting.
Although its diagnostic accuracy for FRI appears to be slightly lower, FDG-PET/CT has several advantages compared to WBC scintigraphy with SPECT/CT. There is only one imaging time-point necessary, and the combination of its high spatial resolution with contrast enhanced $\mathrm{CT}$ (in the newer camera systems even with artifact reduction reconstructions) makes visualizing small lesions possible. The main limitation is that FDG-PET/CT is unable 
Fig. 8 Case 2, Antibiotic cement spacer

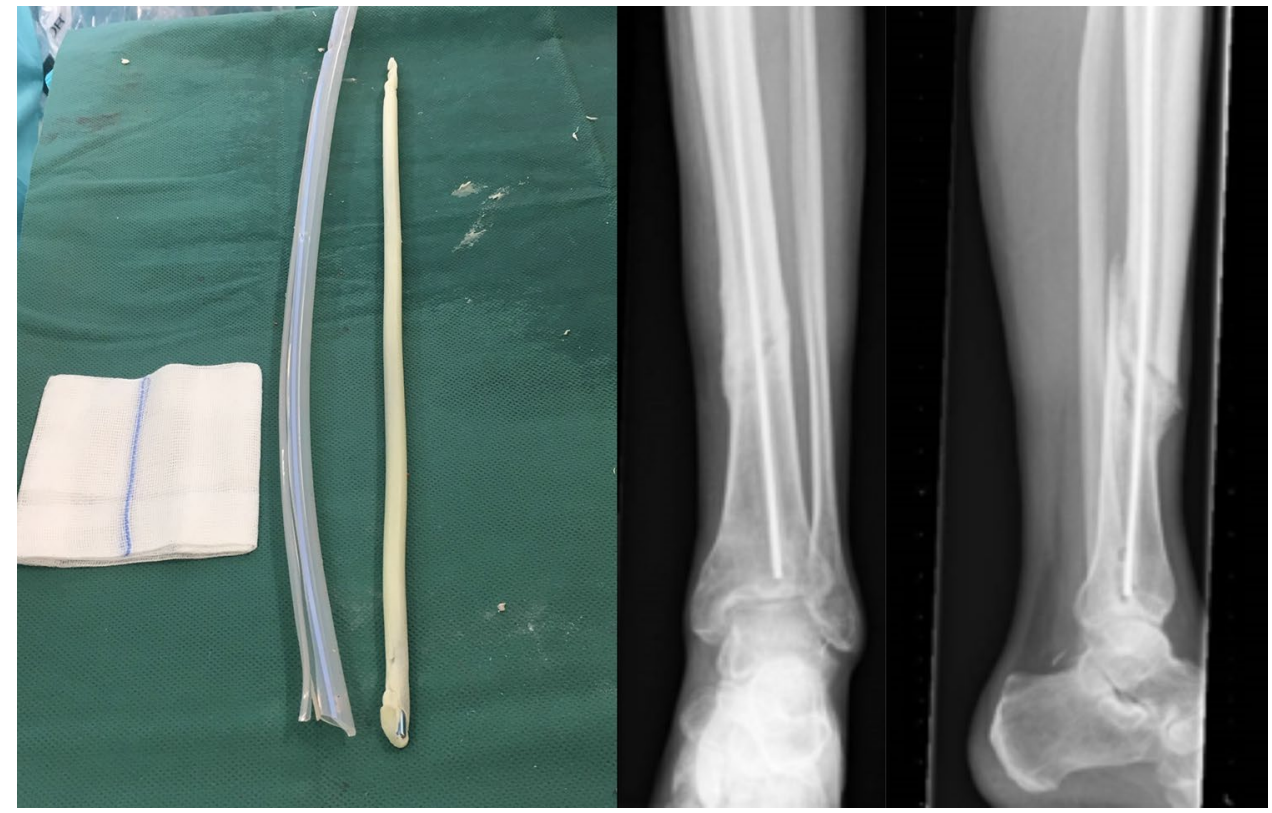

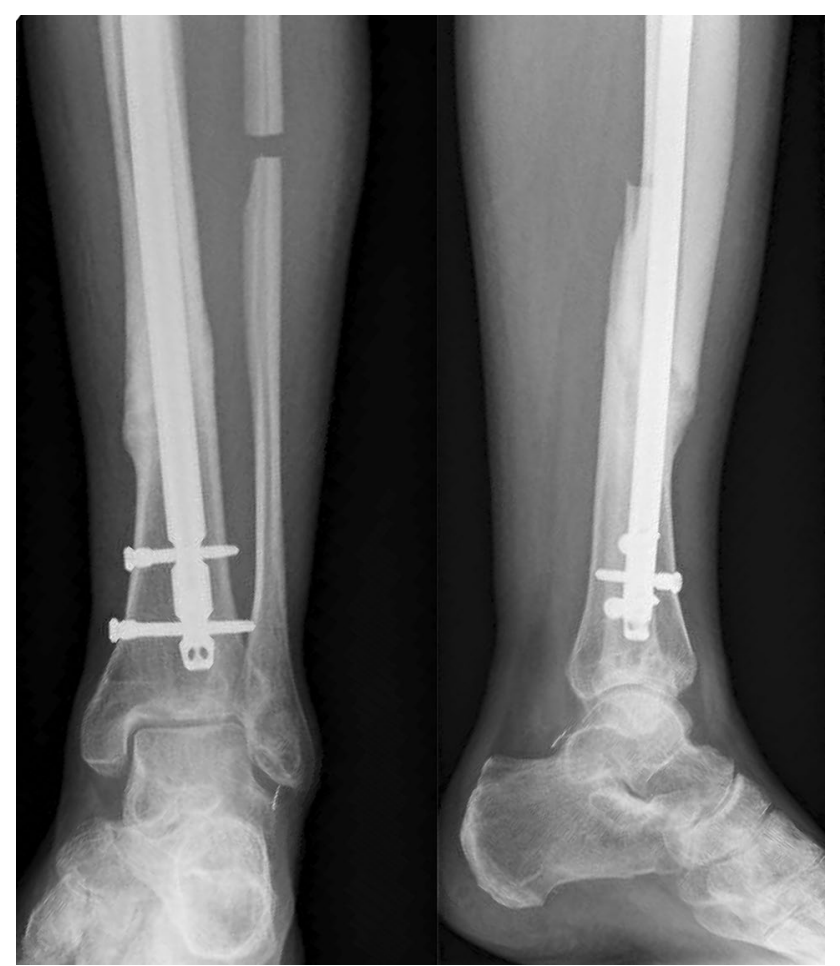

Fig. 9 Case 2, Definitive antibiotic coated osteosynthesis

to differentiate between inflammatory and infectious tissue, which makes discrimination between the two difficult. This is especially problematic in FRI, since inflammation in the first weeks after fracture fixation surgery can be quite common. WBC scintigraphy has a better capacity to distinguish between infection and inflammation, partly due to its dual time-point imaging. Increase of WBC uptake over time correlates with infection and decrease with inflammation.

Recent studies show promising results for the use of nuclear imaging techniques in FRI and are therefore incorporated as a suggestive criterion in a recent update of the consensus criteria [18]. However, before they can be formally accepted as a confirmatory sign, the results of these retrospective studies need to be verified in prospective multicenter trials. Recently, the study protocol for the 'Imaging in Fracture-related Infection (IFI) study' was published [19]. The inclusion for this prospective, multicenter study started in 2019, and will compare the accuracy of MRI, WBC scintigraphy with SPECT/CT and FDG-PET/CT in patients with suspected FRI. The results will be expected within a few years.

\section{Case examples}

\section{Case 1}

A 35-year-old patient with a history of smoking sustained a crural fracture after a bicycle accident and was initially treated with plate osteosynthesis of the tibia and fibula (Fig. 1a+b). After 4 months, the patient was referred to our clinic with an infected non-union of the tibia, extensive scar tissue, and multiple fistulae on the medial side of the lower leg (Fig. 1c). Although confirmatory signs of FRI were present, nuclear imaging was performed to assess the extent of the infection. Both FDG-PET/CT and WBC scintigraphy were performed for scientific research purposes. FDG-PET/ CT showed an increased uptake around the medial malleolus 
and at the non-union site in the distal tibia (Fig. 2, left side). WBC scintigraphy with SPECT and dual time-point imaging showed increased uptake over time in the distal tibia, suggesting a fracture-related infection (Fig. 2, right side).

Surgery was indicated, consisting of a two-stage revision procedure, known as Masquelet procedure. The fistulae and the infected plate were removed (Fig. 3a). Subsequently, the affected bone was debrided (Fig. 3b). The bone defect was temporarily filled with a cement spacer (Fig. 3c). A free myo-cutaneous flap (latissimus dorsi) was used to cover the soft-tissue defect (Fig. 3d). Tissue cultures demonstrated staphylococcus epidermidis, enterococcus faecalis, and staphylococcus capitis for with the patient was treated with amoxicillin and cotrimoxazole for 3 months. After 8 weeks, the second stage of the Masquelet procedure was performed (Fig. 4a). The free flap was lifted, the pseudo-membrane surrounding the cement spacer was opened (Fig. 4b), the spacer was removed, the defect was filled with an autologous cancellous bone graft (Fig. 4c), and the pseudo-membrane was subsequently closed (Fig. 4d).

A postoperative CT-scan demonstrated the tibial bone defect which is filed with the bone graft (Fig. 5, left side). The patient is still in follow-up with no signs of recurrent infection and bone healing is still in progress (Fig. 5, right side).

\section{Case 2}

A 57-year-old man sustained a crural fracture after a scooter accident and was treated with an intramedullary nail. The nail had to be removed after 1 year due to a fracture-related infection (Fig. 6a). He was transferred to our department for further treatment. An MRI scan demonstrated an intramedullary abscess (Brodies abscess) and a non-union of the tibia
(Fig. 6b). Clinical examination showed several fistulae of the lower leg (Fig. 6c).

Both FDG-PET/CT and WBC scintigraphy were performed for scientific research purposes. PET/CT demonstrated increased uptake at the level of the non-union, which was suspect for an FRI (Fig. 7, left side). WBC scintigraphy showed an increased uptake (ratio 1.5 after $24 \mathrm{~h}$ ), which was suspect for FRI as well (Fig. 7, right side).

A re-operation with reaming, wash-out of the medullary canal, and insertion of a temporal cement spacer (prevot nail with antibiotic cement coating) was performed (Fig. 8). The culture results demonstrated a staphylococcus aureus for which the patient was treated with moxifloxacin and rifampicin for 6 weeks. After 6 weeks, the cement spacer was removed, the medullary canal was washed out again and a definitive antibiotic coated nail was inserted. Intraoperative cultures were negative. There were no signs of recurrent infection at follow-up and some callus formation occurred after 3 months (Fig. 9).

\section{Case 3}

A 56-year-old man sustained a subtrochanteric fracture after a fall and was initially treated with a gamma nail (Fig. 10a and b). After 1 year of follow-up, no union of the bone occurred (Fig. 10c). A re-operation with debridement of the pseudarthrosis, a cancellous bone graft, and plate fixation was performed (Fig. 11a). Cultures of the intraoperatively obtained tissue samples were negative. A year later, still no healing of the fracture was present, and the proximal screws started to break (Fig. 11b). Clinical examination demonstrated a normal postoperative scar on the right hip without clinical signs of an infection (Fig. 13a). FDG-PET/CT demonstrated increased uptake in and around the non-union site at the proximal femur, which was suspect for an FRI
Fig. 10 Case 3, Initial fracture and fixation
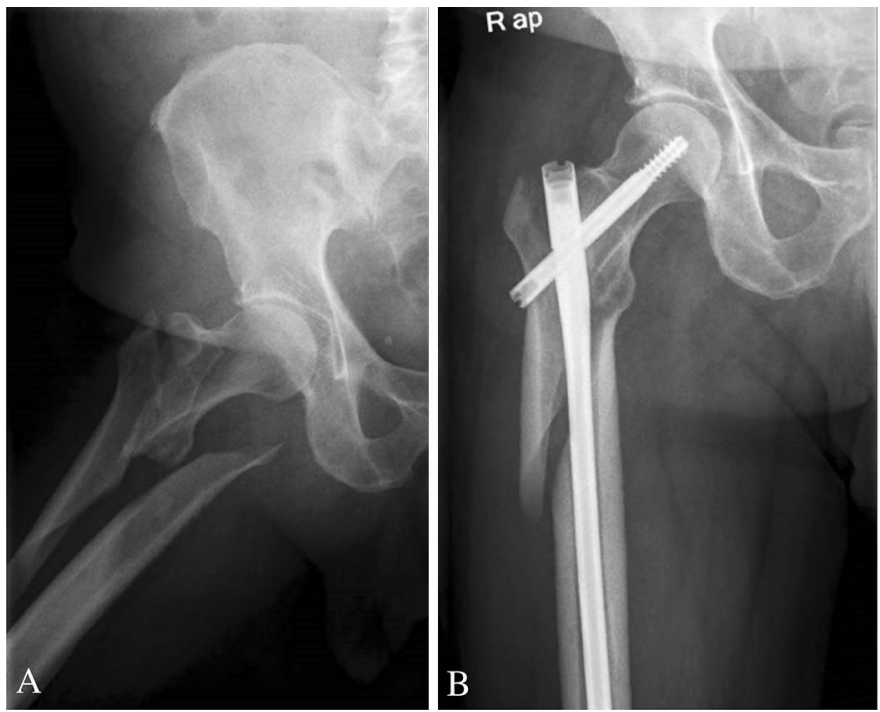


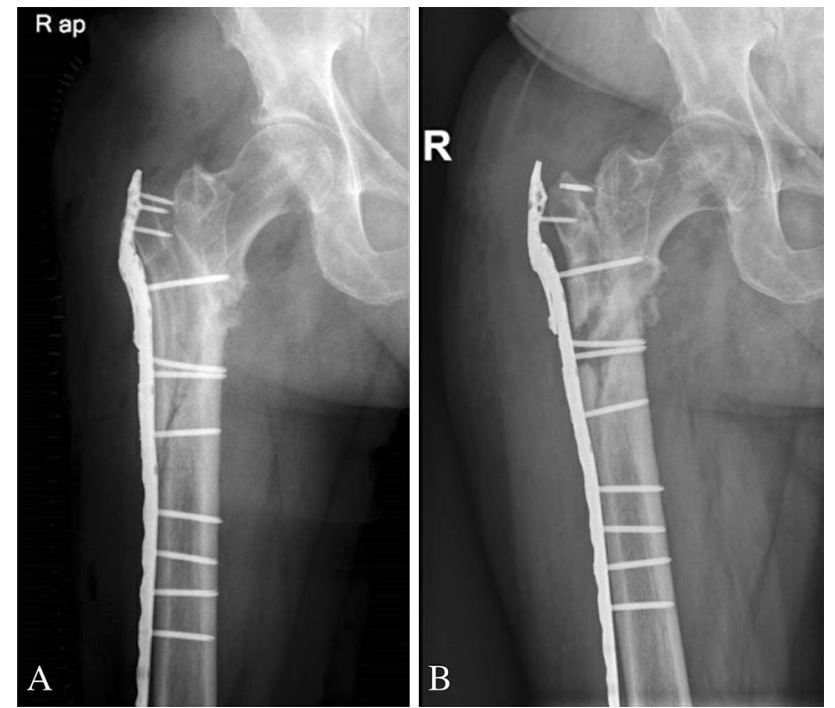

Fig. 11 Case 3, Presentation with implant failure
(Fig. 12). A second re-operation with removal of the plate, debridement of the pseudarthrosis, application of a cancellous bone graft, and fixation with a new DCS-plate was performed. No intra-operative signs of an infection were present (Fig. 13b and c). Immediate postoperative antibiotics (iv cefuroxim and vancomycine for 2 weeks) were started out of precaution based on the positive PET findings. Against all clinical expectations, cultures results demonstrated a staphylococcus epidermidis for which the patient was treated with minocycline for 12 weeks. There were no signs of recurrent infection at follow-up and some fracture healing occurred after 3 months (Fig. 14). This case demonstrates the importance of nuclear imaging in suspected FRI, since infection can be present even without clinical signs on physical examination or during surgery.

\section{Discussion}

Fracture-related infection remains one of the most challenging complications in orthopedic-trauma surgery. There is an invaluable need for a non-invasive diagnostic modality, since

Fig. 12 Case 3, FDG-PET

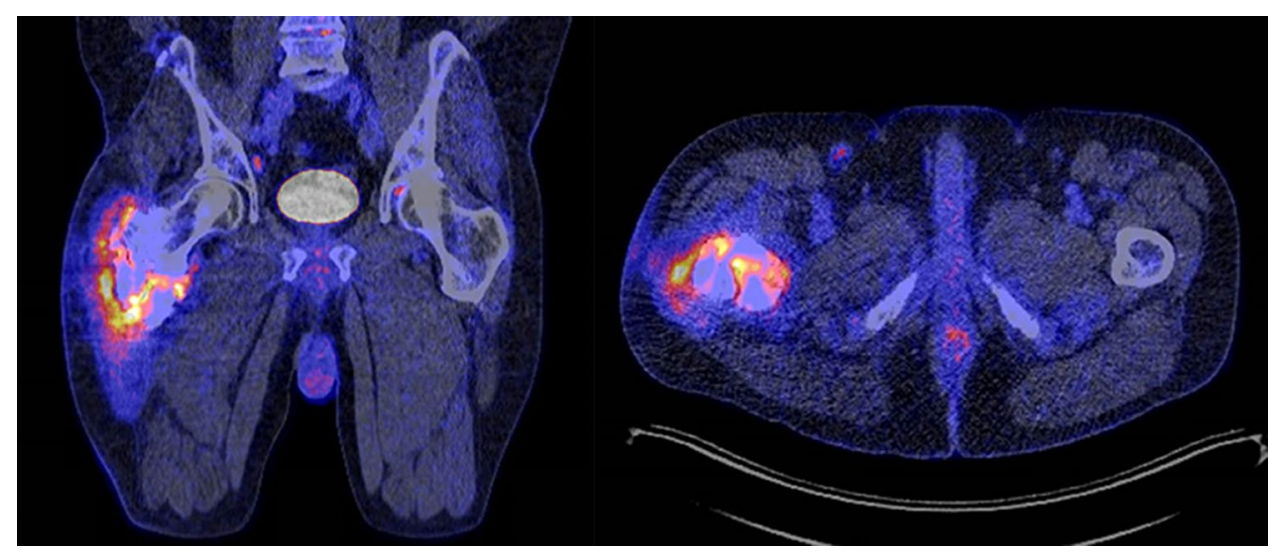

Fig. 13 Case 3, Intra-operative images
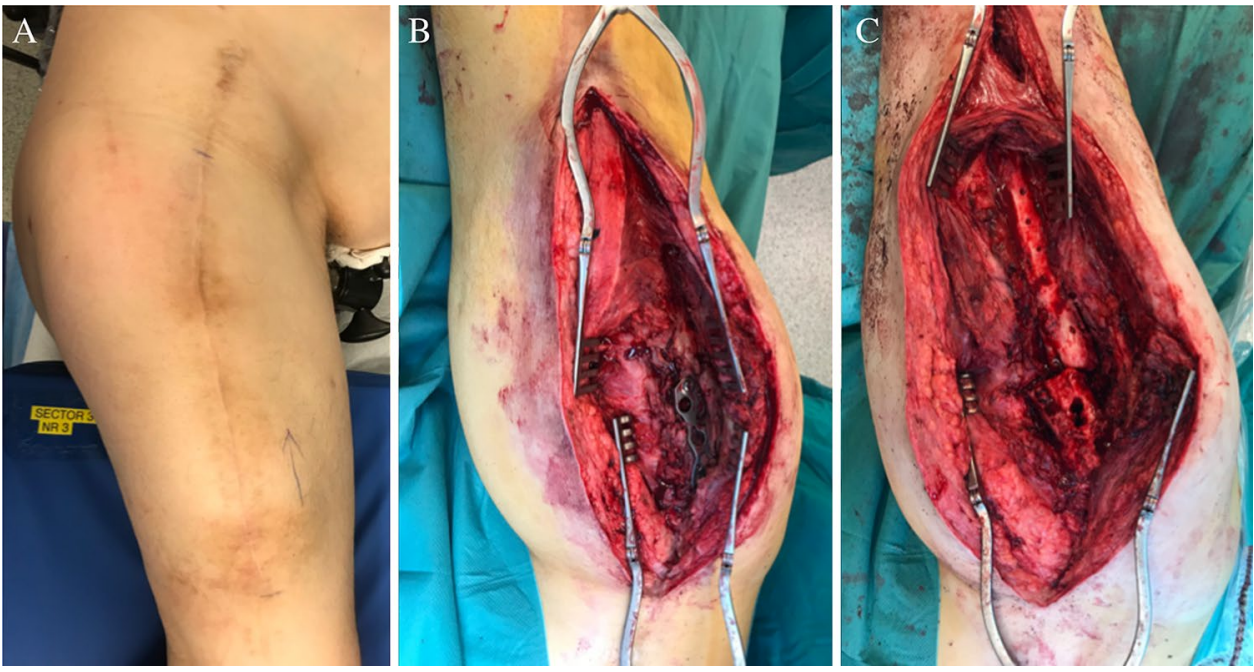


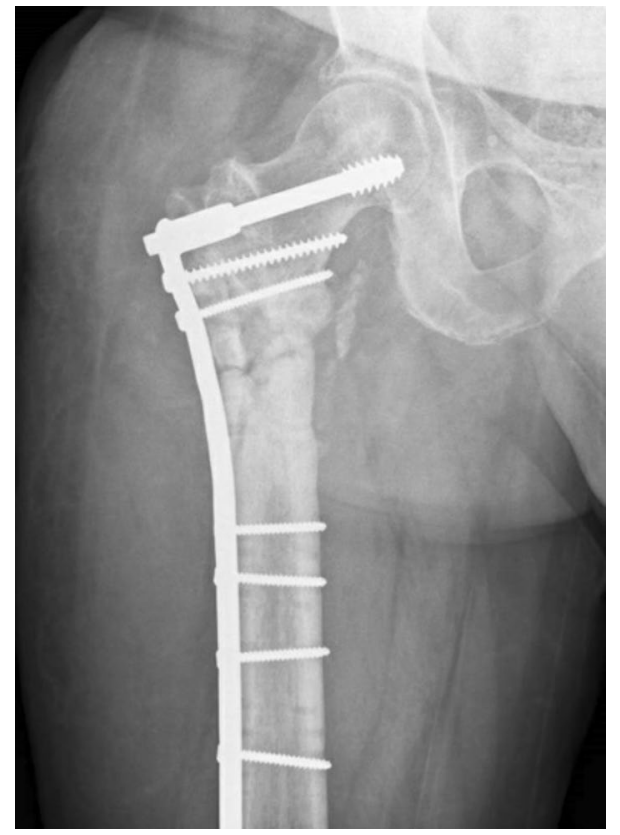

Fig. 14 Case 3, Follow up

early diagnosis and determining the extent of the infection are essential for successful treatment. The treatment consists of proper operative debridement, carefully obtained deep cultures, a wash-out, adequate soft-tissue coverage, stable fixation of the fracture fragments, and tailored antibiotic treatment. Whether an infective implant should be removed or can be retained depends on the duration that an FRI is present (biofilm formation) and the degree of fracture healing.

The recently published consensus definition for FRI is one of the first important steps in standardizing the research and formulating universal diagnostic guidelines [8]. Recent studies on nuclear imaging modalities in diagnosing FRI show promising results. Both WBC scintigraphy with SPECT and FDG-PET/CT show good diagnostic accuracy (0.92 vs 0.83 ) for FRI. WBC scintigraphy has a slightly better diagnostic accuracy, but FDG-PET/CT has several logistical advantages and is generally more widely available. Furthermore, future improvements in PET camera systems will continue to increase its spatial accuracy. It may, therefore, be possible that FDG-PET/CT will surpass the accuracy of WBC scintigraphy in the near future. For now, it is important to verify the results of the recently published retrospective studies in prospective multicenter trials. A prospective, multicenter study (IFI study) comparing MRI, FDG-PET/CT, and WBC scintigraphy with SPECT in FRI is currently conducted.

\section{Compliance with ethical standards}

Conflict of interest None of the authors have a conflict of interest.
Open Access This article is licensed under a Creative Commons Attribution 4.0 International License, which permits use, sharing, adaptation, distribution and reproduction in any medium or format, as long as you give appropriate credit to the original author(s) and the source, provide a link to the Creative Commons licence, and indicate if changes were made. The images or other third party material in this article are included in the article's Creative Commons licence, unless indicated otherwise in a credit line to the material. If material is not included in the article's Creative Commons licence and your intended use is not permitted by statutory regulation or exceeds the permitted use, you will need to obtain permission directly from the copyright holder. To view a copy of this licence, visit http://creativecommons.org/licenses/by/4.0/.

\section{References}

1. Bose D, Kugan R, Stubbs D, McNally M (2015) Management of infected nonunion of the long bones by a multidisciplinary team. Bone Jt J 97B(6):814-817

2. Metsemakers WJ et al (2016) Infection after fracture fixation: current surgical and microbiological concepts. Injury 49(3):511-522

3. Metsemakers WJ, Smeets B, Nijs S, Hoekstra H (2017) Infection after fracture fixation of the tibia: analysis of healthcare utilization and related costs. Injury 48(6):1204-1210

4. Korim MT, Payne R, Bhatia M (2014) A case-control study of surgical site infection following operative fixation of fractures of the ankle in a large UK trauma unit. Bone Jt J 96B(5):636-640

5. Kortram K et al (2017) Risk factors for infectious complications after open fractures; a systematic review and meta-analysis. Int Orthop 41(10):1965-1982

6. McNally M, Nagarajah K (2010) Osteomyelitis. Orthop Trauma 24(6):416-429

7. Trampuz A, Zimmerli W (2006) Diagnosis and treatment of infections associated with fracture-fixation devices. Injury 37:S59-66

8. Metsemakers WJ et al (2018) Fracture-related infection: a consensus on definition from an international expert group. Injury 49:505-510

9. Goebel M et al (2007) Diagnosis of chronic osteitis of the bones in the extremities. Relative value of F-18 FDG-PET. Der Unfallchirurg 110(10):859-866

10. Kaim A et al (2000) Chronic post-traumatic osteomyelitis of the lower extremity: comparison of magnetic resonance imaging and combined bone scintigraphy/immunoscintigraphy with radiolabeled monoclonal antigranulocyte antibodies. Skeletal Radiol 29:378-386

11. Govaert GAM et al (2017) Accuracy of diagnostic imaging modalities for peripheral post-traumatic osteomyelitis-a systematic review of the recent literature. Eur J Nucl Med Mol Imaging 44:1393-1407

12. Ballani NS et al (2007) The value of quantitative uptake of (99 m) Tc-MDP and (99 m)Tc-HMPAO white blood cells in detecting osteomyelitis in violated peripheral bones. J Nucl Med Technol 35(2):91-95

13. Glaudemans AWJM et al (2019) Consensus document for the diagnosis of peripheral bone infection in adults: A joint paper by EANM, EBJIS, and ESR (with ESCMID endorsement). Eur J Nucl Med Mol Imaging 46:957-970

14. Lemans JVC et al (2019) The diagnostic accuracy of ${ }_{18} \mathrm{~F}-\mathrm{FDG}$ PET/ $\mathrm{CT}$ in diagnosing fracture-related infections. Eur J Nucl Med Mol Imaging 46:999-1008

15. Sollini $\mathrm{M}$ et al (2019) ${ }_{18}$ FDG PET/CT in non-union: improving the diagnostic performances by using both PET and CT criteria. Eur J Nucl Med Mol Imaging 46:1605-1615

16. Govaert GAM et al (2018) High diagnostic accuracy of white blood cell scintigraphy for fracture related infections: results of a large retrospective single-center study. Injury 49:1085-1090

17. Rubello D et al (2004) Role of anti-granulocyte Fab' fragment antibody scintigraphy (LeukoScan) in evaluating bone infection: 
acquisition protocol, interpretation criteria and clinical results. Nucl Med Commun 25:39-47

18. Govaert GAM et al (2020) Diagnosing fracture related infection: current concepts and recommendations. J Orthop Trauma 34:8-17

19. Govaert GAM et al (2019) The accuracy of diagnostic Imaging techniques in patients with a suspected Fracture-related Infection (IFI) trial: study protocol for a prospective multicenter cohort study. BMJ Open 9(9):e027772
Publisher's Note Springer Nature remains neutral with regard to jurisdictional claims in published maps and institutional affiliations. 